Results: IIM patients had visible tracer uptake in the skeletal muscles of the extremities. The muscular ${ }^{99 m}$ Tc-PYP uptake was significantly higher in upper limbs of patients than the uptake in the same muscle groups in healthy controls (uptake[biceps] 0.46 vs. $0.33 \mathrm{~g} \mathrm{~mL}^{-1}, \mathrm{p}=0.01$; uptake[triceps] 0.40 vs. $0.27 \mathrm{~g}$ $\left.\mathrm{mL}^{-1}, \mathrm{p}=0.003\right)$. The ${ }^{99 \mathrm{~m}} \mathrm{Tc}$-PYP uptake tended to be higher in the lower limbs of patients than in the lower limbs of controls (uptake[quadriceps] 0.59 vs. 0.48 $\mathrm{g} \mathrm{mL}^{-1}, \mathrm{p}=0.06$ ). The muscular FDG uptake was significantly higher in patients than in controls in both upper limbs (SUV mean [biceps] 1.35 vs. $0.72 \mathrm{~g} \mathrm{~mL}^{-1}$, $\mathrm{p}=0.006$; $\mathrm{SUV}_{\text {mean }}$ [triceps] 0.91 vs. $0.44 \mathrm{~g} \mathrm{~mL}^{-1}, \mathrm{p}=0.0008$ ) and lower limbs ( $S U V_{\text {mean }}$ [quadriceps] 0.84 vs. $0.62 \mathrm{~g} \mathrm{~mL}^{-1}, \mathrm{p}=0.0001$ ). The muscular FDG uptake values were consequently higher than the ${ }^{99 m}$ Tc-PYP uptake values, although not by a constant factor.

Conclusions: Quantitative ${ }^{99 \mathrm{~m}} \mathrm{TC}$-PYP SPECT/CT as well as ${ }^{18} \mathrm{~F}$-FDG PET/CT imaging revealed muscular inflammation in patients with newly onset, untreated IIM. Patients had higher tracer uptake in skeletal muscles groups than healthy controls. Quantification of muscular tracer uptake with the potential to objectively distinguish physiology from pathophysiology could be a valuable tool in the challenging diagnosis of IIMs.

Disclosure of Interest: None declared

DOI: 10.1136/annrheumdis-2017-eular.6000

\section{OP0034 FACTORS ASSOCIATED WITH DISEASE PROGRESSION IN EARLY-DIAGNOSED PULMONARY ARTERIAL HYPERTENSION ASSOCIATED WITH SYSTEMIC SCLEROSIS: LONGITUDINAL DATA FROM THE DETECT COHORT}

C. Mihai ${ }^{1}$, M. Antic ${ }^{2}$, R. Dobrota ${ }^{1,2}$, D. Bondermann ${ }^{3}$, H. Chadha-Boreham ${ }^{4}$ G. Coghlan ${ }^{5}$, C.P. Denton ${ }^{6}$, M. Doelberg ${ }^{4}$, E. Grünig ${ }^{7}$, D. Khanna ${ }^{8}$, V.V. McLaughlin ${ }^{9}$, U. Müller-Ladner ${ }^{10}$, J.E. Pope ${ }^{11}$, D.M. Rosenberg ${ }^{4}$, J.R. Seibold ${ }^{12}$, M.C. Vonk ${ }^{13}$, O. Distler ${ }^{2} .{ }^{1}$ Internal Medicine and Rheumatology, Cantacuzino Hospital, Carol Davila University of Medicine and Pharmacy,

Bucharest, Romania; ${ }^{2}$ Department of Rheumatology, University Hospital Zurich, Zurich, Switzerland; ${ }^{3}$ Department of Internal Medicine II, Division of Cardiology, Medical University of Vienna, Vienna, Austria; ${ }^{4}$ Actelion Pharmaceuticals Ltd, Allschwil, Switzerland; ${ }^{5}$ Cardiology Department; ${ }^{6}$ Centre of Rheumatology, Royal Free Hospital, London, United Kingdom; ${ }^{7}$ Centre for Pulmonary Hypertension, Thoraxclinic, University Hospital Heidelberg, Heidelberg, Germany; ${ }^{8}$ Division of Rheumatology, Department of Medicine; ${ }^{9}$ Division of Cardiology, Department of Medicine, University of Michigan, Ann Arbor, MI, United States; ${ }^{10}$ Department of Rheumatology and Clinical Immunology, Justus-Liebig University, Giessen, Germany; ${ }^{11}$ Division of Rheumatology, Department of Medicine, Western University of Canada, London, Ontario, Canada: ${ }^{12}$ Scleroderma Research Consultants LLC, Litchfield, CT, United States; ${ }^{13}$ Department of Rheumatology, Radboud University Medical Centre, Nijmegen, Netherlands

Background: Pulmonary arterial hypertension (PAH) is a severe complication of systemic sclerosis (SSc).

Objectives: In this longitudinal study we aimed to identify factors associated with an unfavourable outcome in SSc patients with early PAH (SSc-PAH) from the DETECT cohort.

Methods: DETECT enrolled patients with SSc fulfilling the 1980 ACR classification criteria, with a minimal disease duration of 3 years since the first non-Raynaud symptom, and with a lung diffusion capacity for $\mathrm{CO}$ (DLCO) $<60 \%$ of the predicted value. A broad range of clinical and laboratory parameters potentially associated with PAH were assessed, and right heart catheterisation (RHC) was performed in all patients at baseline. Patients diagnosed with PAH were followed up for up to 3 years in centers that agreed for the longitudinal part of the DETECT study, collecting data on survival, World Health Organization (WHO) Functional Class (FC), hospitalization, and PAH-specific treatment. Disease progression was defined as the occurrence of any of the following: WHO-FC worsening, PAH therapy with a drug combination, hospitalization, or death. Associations between baseline variables and disease progression were assessed by univariable logistic regression.

Results: Of the 145 SSc patients with PH enrolled in DETECT, 87 patients were diagnosed with PAH, of whom 57 participated in the longitudinal study (median follow-up time 12.6 months, interquartile range 10.7-21.7 months). Among these 57 patients, $33 / 57(57.9 \%)$ had mild PAH, in WHO FC I or II. During follow-up, 25/57 (43.9\%) patients had disease progression (4 deaths, 11 hospitalizations for $\mathrm{PAH}, 14$ with worsening in WHO FC, and 8 received PAH-specific combination treatment), with a 1-year survival rate of $93 \%$. The following factors [odds ratio, (95\% confidence interval)] were associated with disease progression: male gender [4.1 (1.2-14.1)], high Forced Vital Capacity (FVC) \% predicted/ DLCO \% predicted ratio [3.6 (1.2-10.7)], and high Borg dyspnoea index [1.7 (1.1-2.6)]. Low DLCO (\% predicted) was also significantly associated with progression [area under the curve $(95 \% \mathrm{Cl}) 0.8(0.6-0.9)]$, but the relationship was not linear.

Conclusions: More than $40 \%$ of early-diagnosed SSc-PAH patients in the DETECT cohort who were followed over time had disease progression during a rather short follow-up time, with male gender, functional capacity, and pulmonary function tests (low DLCO, high FVC\%/DLCO \% predicted ratio) at PAH diagnosis being associated with progression. This suggests that even mild and early detected PAH should be regarded as a high-risk complication of SSc, and every effort to make an early diagnosis is valuable.

Acknowledgements: The authors thank all investigators and patients involved in
DETECT.

Disclosure of Interest: C. Mihai Grant/research support from: Actelion, Geneva Romfarm, Abbvie, Speakers bureau: Actelion, Geneva-Romfarm, RocheGenentech, M. Antic: None declared, R. Dobrota Grant/research support from: Pfizer, Actelion, Speakers bureau: Actelion, D. Bondermann Consultant for: Actelion, GSK, MSD, Bayer, Pfizer, AOP Orphan, United Therapeutics, H. Chadha-Boreham Employee of: Actelion, G. Coghlan Consultant for: Actelion, GSK, Bayer, United Therapeutics, Endotronics, Speakers bureau: Actelion, GSK, Bayer, United Therapeutics, C. Denton Consultant for: Actelion, M. Doelberg Employee of: Actelion, E. Grünig Grant/research support from: United Therapeutics, Actelion, MSD/Bayer, GSK, Consultant for: United Therapeutics, Actelion, MSD/Bayer, GSK, Speakers bureau: United Therapeutics, Actelion, MSD/Bayer, GSK, D. Khanna Grant/research support from: Bayer, BMS, Genentech/Roche, Sanofi-Aventis, NIH K24AR063120, Consultant for: Actelion, Bayer, Covis, Cytori, EMD Serono, Genentech/Roche, Gilead, GSK, Sanofi-Aventis, V. McLaughlin Consultant for: Actelion, U. Müller-Ladner Consultant for: Actelion, Speakers bureau: Actelion, J. Pope Consultant for: Actelion, Bayer, BMS, Merck, Roche, D. Rosenberg Employee of: Actelion, J. Seibold Consultant for: Acer, Actelion, Anthera, arGen-X, aTyr, Bayer, Blade, Boehringer-Ingelheim, Bristol Myers Squibb, Biogen Idec, Covis, Eiger, EMD Serono, Genkyotex, Gilead, Ironwood, Medac, Medlmmune, Mitsubishi, Octapharma, Roche-Genentech, Sanofi, Teva and United Therapeutics., M. Vonk Consultant for: Actelion, O. Distler Grant/research support from: 4 D Science, Actelion, Active Biotec, Bayer, Biogen Idec, Boehringer Ingelheim Pharma, BMS, ChemomAb, EpiPharm, Ergonex, espeRare foundation, GSK, Roche-Genentech, Inventiva, Lilly, medac, Medlmmune, Mitsubishi Tanabe, Pharmacyclics, Pfizer, Sanofi, Serodapharm and Sinoxa, Consultant for: $4 \mathrm{D}$ Science, Actelion, Active Biotec, Bayer, Biogen Idec, Boehringer Ingelheim Pharma, BMS, ChemomAb, EpiPharm, Ergonex, espeRare foundation, GSK,Roche-Genentech, Inventiva, Lilly, medac, Medlmmune, Mitsubishi Tanabe, Pharmacyclics, Pfizer, Sanofi, Serodapharm and Sinoxa DOI: 10.1136/annrheumdis-2017-eular.2866

\section{OP0035 PRELIMINARY ANALYSIS OF NAILFOLD CAPILLAROSCOPY IN THE VERY EARLY DIAGNOSIS OF SYSTEMIC SCLEROSIS (VEDOSS): THE CAPI-VEDOSS EXPERIENCE}

M. Cutolo ${ }^{1}$, V. Smith 2,3 , O. Distler ${ }^{4}$, O. Kowal-Bielecka ${ }^{5}$, Y. Allanore ${ }^{6}$,

M. Matucci-Cerinic ${ }^{7}$ on behalf of EUSTAR coworkers. ${ }^{1}$ Research Laboratory and Academic Unit of Clinical Rheumatology, Department of Internal Medicine, University of Genova, Genova, Italy; ${ }^{2}$ Department of Internal Medicine, University of Ghent; ${ }^{3}$ Department of Rheumatology, Ghent University Hospital, Ghent, Belgium; ${ }^{4}$ Department of Rheumatology, University Hospital Zurich, Zurich, Switzerland; ${ }^{5}$ Department of Rheumatology and Internal Medicine, Medical University of Bialystok, Bialystok, Poland; ${ }^{6}$ Department of Rheumatology, Paris Descartes University, Cochin Hospital, Paris, France: ${ }^{7}$ Department of Experimental and Clinical Medicine, Division of Rheumatology, University of Florence, Florence, Italy

Background: In Systemic Sclerosis (SSc), before the onset of clinical signs of fibrosis, puffy fingers, specific autoantibodies and microcirculatory modifications are present and are identified in the VEDOSS criteria for "(very) early" disease. No large scale studies are available indicating the prevalence of nailfold videocapillaroscopic (NVC) SSc patterns or quantitated capillaroscopic characteristics in a "(very) early" cohort.

Objectives: Evaluation of the prevalence of SSc patterns and quantitated capillaroscopic characteristics in a "(very) early" crossectional SSc cohort.

Methods: Multicentre observational cohort study of patients with two strata (Figure): 1.Raynaud's phenomenon (RP), Anti Nuclear Antibody (ANA) positive $(+)$, 2. RP+, ANA negative (-). "Target" (RP+, ANA+, puffy finger $[\mathrm{PF}]+/-$, SSc- antibody (SSc-AB)+, NVC+) and "control" (RP+, ANA-, SSc-AB-, NVC-) subsets were described. NVC patterns (normal/non-specific alterations; nonspecific abnormalities; SSc-patterns ("Early", "Active";"'Late"; "Sclerodermalike") and quantitative ("absent (="none" or "rare") and "present" (="moderate" or "extensive") capillaroscopic alterations for giants, haemorrhages, capillary loss and abnormally shaped (=bushy capillaries) were evaluated). Generalized Estimating Equations (GEE) were used to assess differences in prevalences of NVC patterns between strata, taking clusters of patients within centers into account.

Results: $1085 \mathrm{RP}$ patients from 40 centres (median number [ ${ }^{\circ}$ ] of pt 12 (minimum [min] 1-maximum [max] 393) per centre were enrolled in the VEDOSS online database. Due to erroneous included/missing data (e.g. erroneous: absence/missing information on RP, presence of former ACR criteria for SSc, skin involvement at baseline; missing info on: ANA positivity, PF, Cap, SScAB) 750 patients (median $n^{\circ}$ of 7 (min:1-max:271) were retained for the analysis. SSc nailfold videocapillaroscopic (NVC) patterns ("Early", "Active", "Late", "Scleroderma like" were present in $79 \%, 13 \%, 0 \%, 8 \%$ ) as well as presence of "moderate" or "extensive" giants $(49 \%)$, hemorrhages $(32 \%)$, capillary loss $(11 \%)$ and abnormal shapes $(11 \%)$ of "target" patients and per definition in $0 \%$ of "controls". Estimated distribution of SSc patterns differed in the ANA+/- stratum: 49\%/15\% (p <0.001) ("Early" 40/13\%; "Active" 5/2\%; "Late" 0/0\%; "Scleroderma like" $3 / 0 \%$ ). For the quantitative capillaroscopic characteristics a statistically significant difference in the presence of "moderate" or "extensive" giants $(23 / 5 \%$, $\mathrm{p}=0.027)$ between the $\mathrm{ANA}+/$ - stratum was found, hemorrhages $(18 / 9 \%, \mathrm{p}=0.200)$, capillary loss $(5 / 2 \%, p=0.798)$ and abnormal (ramified) shapes $(7 / 2 \%, p=0.445)$. 